\title{
Smart search for optimal technological processes in cyber-physical systems
}

\author{
Vasiliy Golovin ${ }^{1, *}$ \\ ${ }^{1}$ Sevastopol State University, Polytechnical Institute, 299053 Sevastopol, Russia
}

\begin{abstract}
Digital production dictates new approaches to the organization of technological processes, including the development of cyber-physical systems within the framework of Industry 4.0. The development of these systems involves the use of not only classical methods, but also additive technologies in production. The article deals with the concept of a smart production system to find the optimal technological process, which is based on the user defined constraints and expert data of the cloud cyber-physical system.
\end{abstract}

\section{Introduction}

Today, by combining achievements in digital technologies such as wireless networks, cloud computing, big data and artificial intelligence on the one hand, and progress in smart materials, nanotechnology and 3D printing on the other, we are around of the beginning of the Fourth industrial revolution. Universal access to the global network and a huge amount of user data open up unlimited opportunities that people could not imagine a few years ago [1]. However, researchers in the scientific community and in industry have recognized that the coming in of the fourth industrial revolution will transform almost all aspects of our modern life: production methods, business models, production management methods, etc. The new skills and retraining of labor resources are required, with the prospect of increasing the share of automation and revision of the future of working professions [2].

Increased computing power, augmented reality and virtual reality systems will be critical in implementation of many smart manufacturing strategies. Integration of these technologies into smart factories is necessary for rapid adaptation of technological innovations in order to produce high-quality products and services.

The implementation of the concept "industry 4.0" provides for the formation of cyberphysical systems (Cyber-Physical Systems - CPS). Such systems combine hardware, process equipment and logistics systems [3].

Automatic allocation of system resources based on input information will be an integral part of CPS for production. The input data includes information about a part's geometry (geometries and part's dimensions) and constraints (quality, mechanical and economic). So far, the research efforts to identify such a distributive element of the system have been focused on the main areas of production, i.e. subtractive and additive technologies. For subtractive production process selection can be carried out by geometric analysis of the

*Corresponding author: vig220@mail.ru 
elements and the subsequent comparison of these elements with the corresponding processing processes [4]. For additive manufacturing, process selection can be made based on material selection, part size and construction quality [5]. This difference in process selection criteria makes it difficult to create and integrate a universal logic distributor into a dynamic and autonomous CPS.

The purpose of this article is to describe the algorithm for finding the optimal production process, which is based on the constraint satisfaction problem (CSP). In particular, the task of selecting a production process in CPS is to find a sequence of technological processes that meet the specified restrictions, which describes the cost and quality of the product (set by the user), and is based on the capabilities of the CPS itself [6]. Since the number of processes and process combinations in CPS is limited, programming in constraints is ideal for handling the task.

\section{Smart process selector}

The implementation of such smart process selector (SPS) will be based on close interaction between the user and the CPS. The following algorithm is proposed: transformation of primary information (a part's geometry) into the space of solutions of possible combinations of production processes; application of user-defined constraints to reduce the created space of solutions based on local deductions.

Thus, the user determines the primary information about the product in the form of its 3D-model, as well as the permissible ranges in quality, mechanical properties and cost. This information is then passed to the CPS, which recognizes the geometry elements and creates a list of possible process options that are required to produce the part. Finally, in order to reduce the number of options in the list, expert knowledge of optimal technological processes and predefined ranges of restrictions are applied in order to form a final list that meets the requirements of both the user and the CPS.

The CSP problem consists of a set of variables $V=\left\{x_{1}, \ldots, x_{n}\right\}$, a set of domains of $D_{i}$ values for each variable $x_{i}$, and a set of constraints and relations. Each value domain is a finite set of values that the corresponding variable can take. The state of the task is determined by assigning values to some or all of these variables $\left\{X_{i}=V_{i}, X_{j}=V_{j}, \ldots\right\}$. An assignment that does not violate any restrictions is valid. A complete assignment is one in which each variable participates, and the solution of the CSP problem is a complete assignment that satisfies all the constraints.

Application of the CSP problem for implementation of SPS is shown on fig 1. First, partial information is generated in the form of a space of possible solutions of technological process sequences based on the input geometric information. Then, the user's limitations (material, build quality, value, etc.) are imposed on this solution space. And, finally, the search for a list of technological process options using expert knowledge about the capabilities of the system is conducted.

Before you can find solutions that satisfy the constraints, you must define the initial task and the search space. The initial task is determined by the user-defined geometric information in the form of a 3D model and a complete list of all production processes available in CPS, grouped by classes (additive, subtractive, deformation, etc.).

Very complex methods of analysis of the geometry for selection of subtractive manufacturing processes already exist, and the methods are able to recognize the signs, the generation of the tool's path on the basis of the recognized signs [6], the analysis of dimensions, tolerance analysis, etc [4]. Similar methods of analysis exist for the selection of additive [5] and deformation processes as well. A list of possible sequences of production process classes can be generated based on the available equipment in the CPS. 


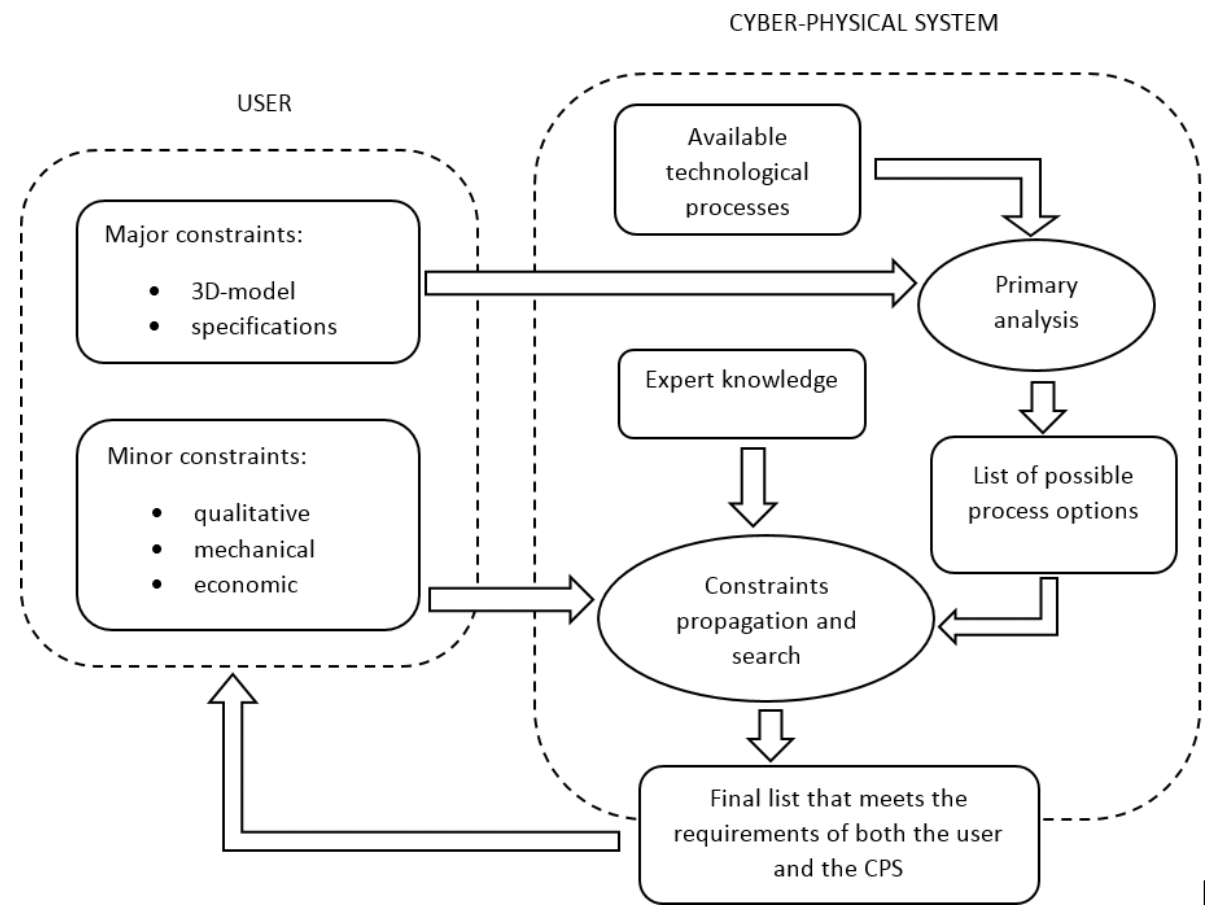

Fig. 1. Constraint satisfaction problem for smart manufacturing.

In the example shown on fig. 2, the initial geometry analysis shows a combination of extrusion and mechanical removal technologies. The solution space of a sequence of processes is generated by selecting the raw material (powder, preform or sheet material), and then defining the physical processes necessary for the production of the geometry with the use of selected raw materials. After this sequence of high-level processes have been identified, the processes within the specific categories are selected from the available CPS.

Taking into consideration the solution space of all the individual process sequences that fall into each of the above process groups, user specifications (qualitative, mechanical, or economic) can later be used as distributors to limit the solution space with local deductions. This requires a quantitative assessment of each option using the criteria specified in table 1. After having the quantitative assessment of all processes, the user can specify a range with a maximum and minimum value for each of the criteria.

Table 1. Criteria for the evaluation of flowsheet options.

\begin{tabular}{|c|l|}
\hline Qualitative & Roughness, tolerances, etc. \\
\hline Mechanical & $\begin{array}{l}\text { Strength of the material, residual } \\
\text { stresses, etc. }\end{array}$ \\
\hline Economic & $\begin{array}{l}\text { Productivity, percentage of } \\
\text { material usage, tool cost, etc. }\end{array}$ \\
\hline
\end{tabular}

Thus, the search spaces for possible processes can be narrowed by eliminating processes that are outside the user-defined range. In this case, user specifications act as distributors that limit the range in the solution space to local deductions, which is a classical problem of logical constraint programming. Such a constraint propagation system can be implemented for production processes by using similar methods based on artificial intelligence that already exist for parametric frameworks in CAD systems. 


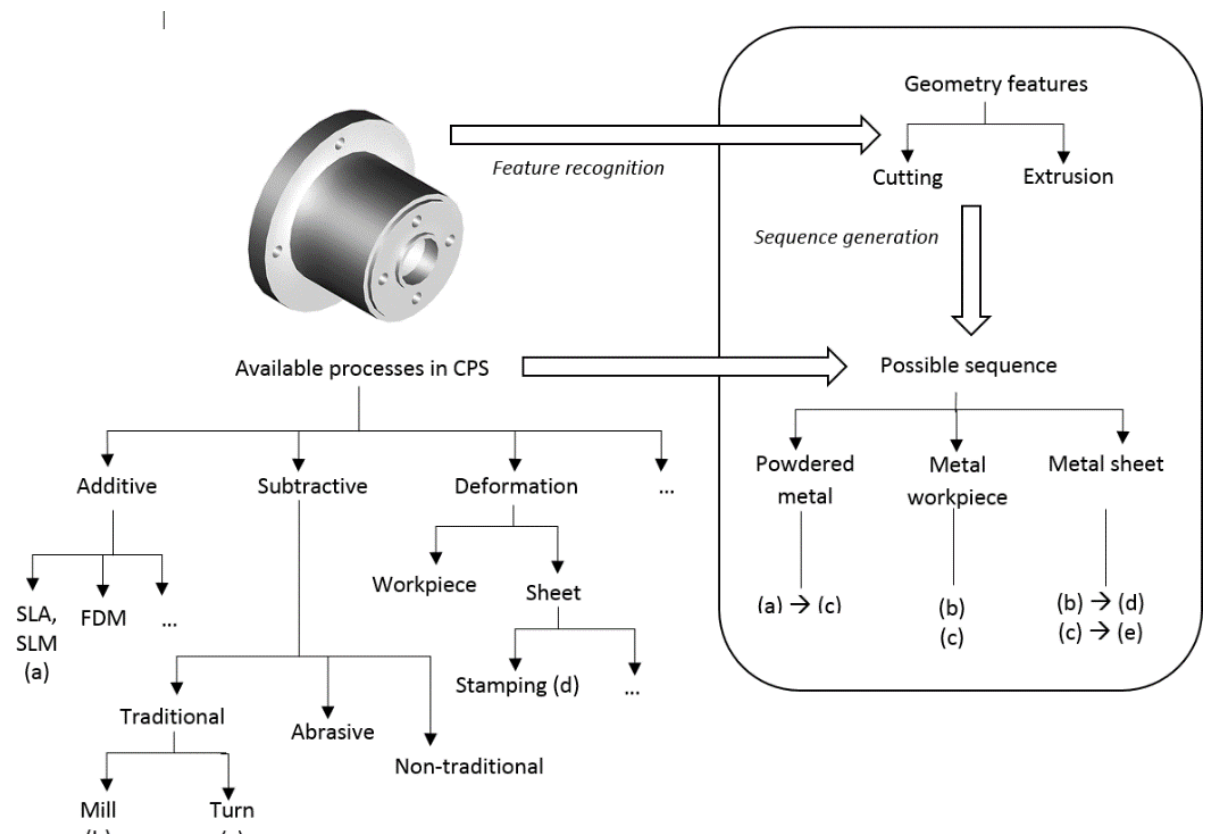

(b)

(c)

Fig. 2. Search for the solution space of the process sequence in the SPS.

The final step in the process selection process is to find the space of all processes that are included in the user-defined ranges of performance criteria to determine the optimal solution for the CPS. At this stage, expert knowledge about CPS, i.e. system-specific information obtained from big data analysis, cost analysis and system constraints that are in the cloud [7], can be used to compile a definitive list of process sequences. In CPS, data for Analytics can be obtained using data collection tools in production processes or directly from other CPS systems [3]. Data on material properties such as porosity, cooling rate, residual stresses, etc., which are difficult to measure directly, can be obtained using numerical simulation methods [6]. Using this data, you can identify models that can detect patterns in process selection for similar geometries that are required to finally find the optimal sequence of the process. Economic information about CPS (operating costs, depreciation, the technological process, etc.), can also be used to select the optimal sequence of processes. A detailed study of increase the speed of production for the group of additive, subtractive and deformation processes showed that it is necessary to take into account such constraints as rigidity of the machine, the acceleration of the actuator, heat transfer and flow of fluids [3]. Finally, restrictions on the CPS itself, such as the physical location of machines, weight and size limits for each production process, machine capabilities, etc., should also be taken into account when looking for the optimal process.

\section{Conclusions}

In the development of cyber-physical systems, particularly Industry 4.0, there is a growing need to define a methodology for intelligent process selection for production systems. This article presents the concept of implementing a smart process selector based on satisfying the limitations laid down at the user level and knowledge about the system itself. In the future, this concept will help to create a cloud-based intelligent manufacturing system with high added value. 


\section{References}

1. K. Schwab, The fourth industrial revolution: crown. (Business, 2017).

2. A.P. Dobrynin, K.YU. CHernyh, V.P. Kupriyanovskij, P.V. Kupriyanovskij, S.A. Sinyagov, International Journal of Open Information Technologies, v.4, №1, pp. 4-11 (2016).

3. A.R. Ingemansson, Naukoyomkie tekhnologii v mashinostroenii, v.1, №.7, pp. 45-48 (2016).

4. J.H. Han, M. Pratt,W.C. Regli, Manufacturing feature recognition from solid models. IEEE Trans Robot Autom (2000).

5. C.G. Mançanares, E. de S. Zancul, J. da Silva Cavalcante, P.A. Cauchick Miguel. Additive manufacturing process selection based on parts' selection criteria. IntJ Adv Manuf Technol, v. 80, Issue 5-8, pp. 1007-1014 (2015).

6. V.A. Levencov, A.E. Radaev, N.N. Nikolaevskij, Nauchnotekhnicheskie vedomosti Sankt-Peterburgskogo gosudarstvennogo politekhnicheskogo universiteta. Ser.: EHkonomicheskie nauki. v.10, №1. (2017).

7. A. Colombo, Bangemann-Go., S. Karnouskos, Industrial Cloud-based cyber-physical Systems. Approach IMC-AESOP, Springer Science \& Business Media (2014). 